\title{
Twist Drill Evacuation of Chronic Subdural Hematoma in Comorbid Patients
}

\author{
Salma R. Abdel-Hamid*, Radwan Nouby, Wael M. A. Al-Ghani, Mohamed A. Ragaee \\ Neurosurgery Department, Assuit University Hospital, Assuit, Egypt \\ Email: *Salmaneuro1991@gmail.com
}

How to cite this paper: Abdel-Hamid, S.R., Nouby, R., Al-Ghani, W.M.A. and Ragaee, M.A. (2019) Twist Drill Evacuation of Chronic Subdural Hematoma in Comorbid Patients. Open Journal of Modern Neurosurgery, 9, 379-392.

https://doi.org/10.4236/ojmn.2019.94035

Received: July 13, 2019

Accepted: September 9, 2019

Published: September 12, 2019

Copyright $\odot 2019$ by author(s) and Scientific Research Publishing Inc. This work is licensed under the Creative Commons Attribution International License (CC BY 4.0).

http://creativecommons.org/licenses/by/4.0/

\begin{abstract}
Chronic Subdural Hematoma (CSDH) is one of the most common types of intracranial hemorrhage, and the prognosis is good if treated properly. The standard treatment for CSDH is surgical evacuation. Various surgical procedures are used such as burr holes evacuation, twist-drill craniostomy, and craniotomy. Our aim is to evaluate the feasibility and safety of twist dill as a first-choice treatment in evacuation of CSDH in comorbid patients. Methods: This study is a prospective analysis of CSDH evacuation using two twist drill craniostomy holes and irrigation conducted on 21 patients with different comorbidities in Assuit University Hospital between May 2017 and May 2018. Results: The overall postoperative clinical improvement was 95.2\%. The operative time was less than 10 minutes in $71.4 \%$ of the patients. 4 patients showed residual collection in the post-operative CT (19\%). Pneumocephaly was detected in 2 patients (9.5\%). Postoperative seizures occurred in 2 patients $(9.5 \%)$, and subarachnoid hemorrhage was detected in one patient (4.7\%). Conclusion: This approach is a new modification of twist drill craniostomy which can decrease the postoperative residual and recurrence rates and also still a minimally invasive and lifesaving technique in patients with different comorbidities.
\end{abstract}

\section{Keywords}

Chronic Subdural Hematoma, Twist Drill Craniostomy, Minimally Invasive Neurosurgery

\section{Introduction}

Chronic subdural hematoma (CSDH) is considered one of the most common types of intracranial hemorrhage. Its incidence increases in elderly population. Early diagnosis and appropriate surgical choice allow complete recovery in most 
cases [1] [2].

Despite general agreement about the indication of operative treatment, the choice of the convenient surgical procedure in CSDH is still controversial [3]. Although burr hole craniotomy is considered as standard surgical treatment for $\mathrm{CSDH}$ still the outcome reported in the literature varies widely with a percentage of reoperation up to $37 \%$ [4].

Twist drill craniostomy is considered one of the minimally invasive and time-saving maneuvers of treatment of CSDH. However, recurrence of the hematoma was one of the most discouraging complications of this procedure. Various modifications were previously done over the classic technique aiming at reducing the complications and improving the surgical morbidity. In this study we treated patients who suffered from different co-morbid conditions e.g. liver failure, renal failure, bleeding tendencies, hypertension... etc. presented by unilateral CSDH after infiltration of a local anesthetic agent by double twist drill craniostomy and irrigation with closed system drainage. There are many modifications that had been added to the classic twist drill craniostomy technique to reduce the risk of compilications and improve the results. In our study, we are discussing the efficacy and safety of double twist drill craniostomy in co-morbid patients as a modification of the classic technique [5] [6].

\section{Patients and Methods}

This prospective clinical trial was carried out on 21 patients from May 2017 to May 2018 in Assuit University Hospital, Egypt. All patients with unilateral symptomatic CSDH were part of our study. Patients with asymptomatic or incidentally discovered hematoma, patients with bilateral CSDH, subacute subdural hematoma or mixed density hematomas, post-surgical CSDH or recurrent hematomas were excluded from the study.

A written informed consent in Arabic was obtained from the patient or from a first degree relative (if the patient is comatose) before being enrolled in the study, explaining the other treatment options, the advantages of the TDC procedure along with its possible complications and the need of further studies to evaluate the postoperative results of the procedure.

Ethical approval was taken from the Institutional Ethics Committee.

Detailed history including history of trauma and medical history was obtained.

Complete clinical examination was performed and neurological assessment was performed using Glasgow Coma Scale (GCS), Markwalder's Neurological Grading System (MGS) and motor power assessment using Medical Research Council Scale (MRC).

All patients were treated at the operative room after partial hair shaving, disinfection and infiltration with local anesthesia. Localization of the surgical points was done (the first point is $1 \mathrm{~cm}$ anterior to the coronal suture and the second point is in the parietal region, modified according to the radiological site of the hematoma) as shown in Figure 1. 


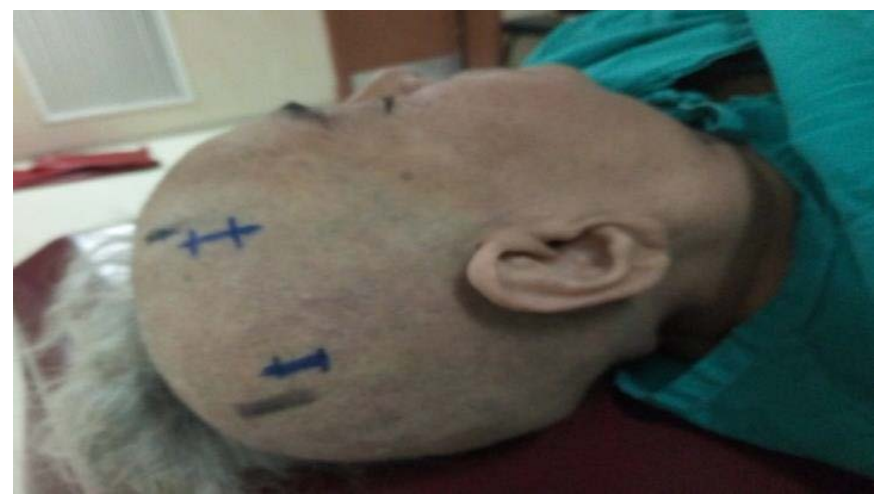

Figure 1. Pre-operative picture showing the patient after being positioned and localizing the sites of the double twist drill craniostomy holes.

Skin incisions were done of approximately $0.5 \mathrm{~cm}$ in radius. Twist drill was performed using a $5 \mathrm{~mm}$ hand driven drill at 45-degree angle to the skull (Figure 2). The dura was opened by the drill itself in most of the cases, in other cases diathermy cauterization was needed to open the dura. Efflux of the hematoma is then allowed to occur passively (with avoidance of rapid decompression). Irrigation using sterile warm saline was done until the out coming fluid is clear. Subcutaneous suction drain was then inserted in dependent position and the wound is closed (Figure 3).

All patients are admitted to the ICU for close observation and monitoring of the neurological conditions for 24 hours, the patients lie flat for the first 24 hours postoperative and mobilization is started on the next day.

Then the patient is transported to the general ward for another $24-48$ hours if improved. Suction drain is usually removed after 48 hours.

Postoperative anti-epileptics and analgesics are administered to all patients. Anti-epileptics are continued for at least 1 to 3 months then gradually tapered. Sutures are removed on the $10^{\text {th }}$ postoperative day.

We evaluated the patients clinically and radiologically with C.T brain after 2 weeks of the surgery to confirm the complete evacuation of the hematoma and brain re-expansion which would not be clear in immediate post-operative imaging (Figure 4 and Figure 5). The patients are followed up to 6 months postoperatively.

We classified the studied variable in our study as following:

1) Primary outcome: Operative time is the primary studied outcome in our study. Time calculation starts instantly on entering of the patient to the operative room and also timing is started with the start of the surgical steps.

\section{2) Secondary outcomes:}

\section{Clinical outcome:}

Neurological improvement determined by the patient's level of conscious and neurological status.

Functional improvement is rated by Markwalder Grading Score:

Grade 0 : Neurologically normal. 


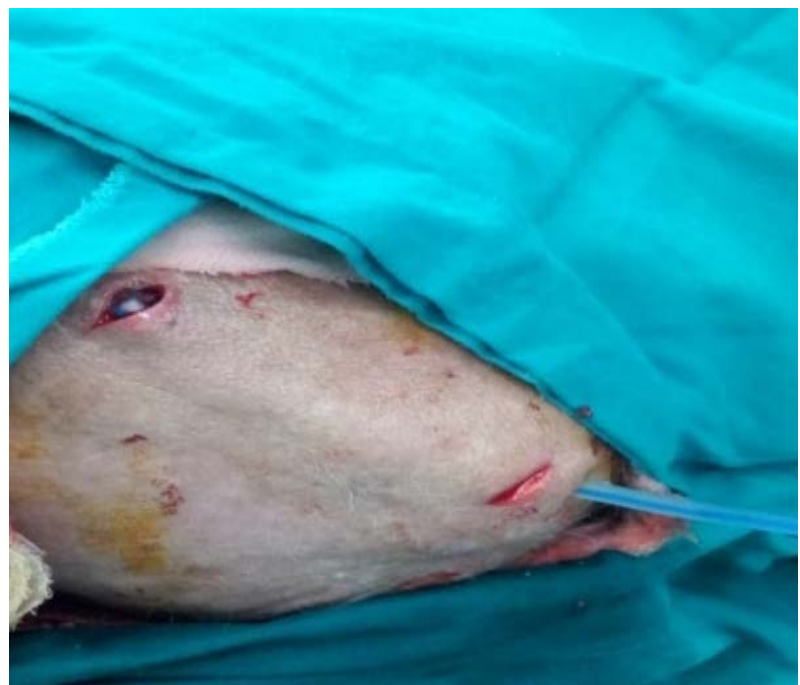

Figure 2. Showing the double craniostomy wounds after evacuation of the hematoma and insertion of the subcutaneous drain.

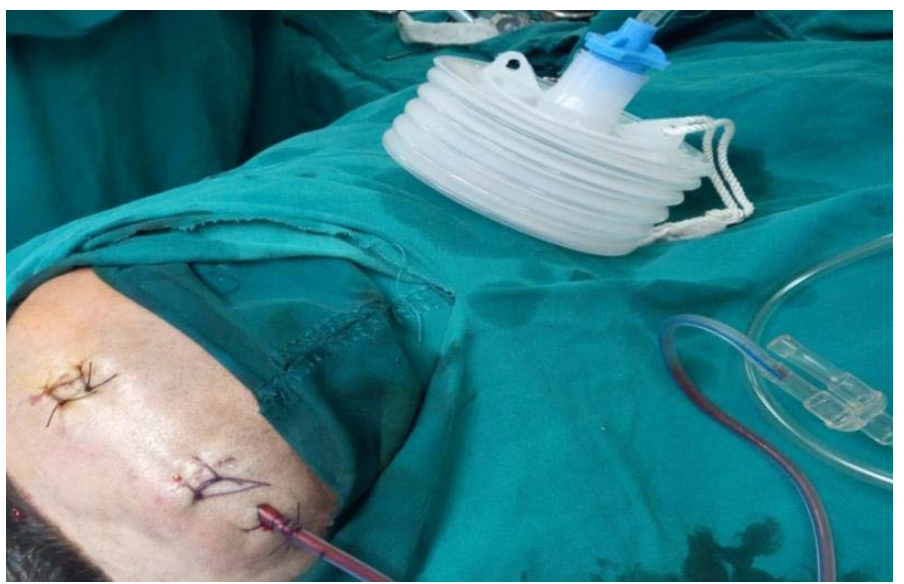

Figure 3. Intra operative picture after small wound closure.

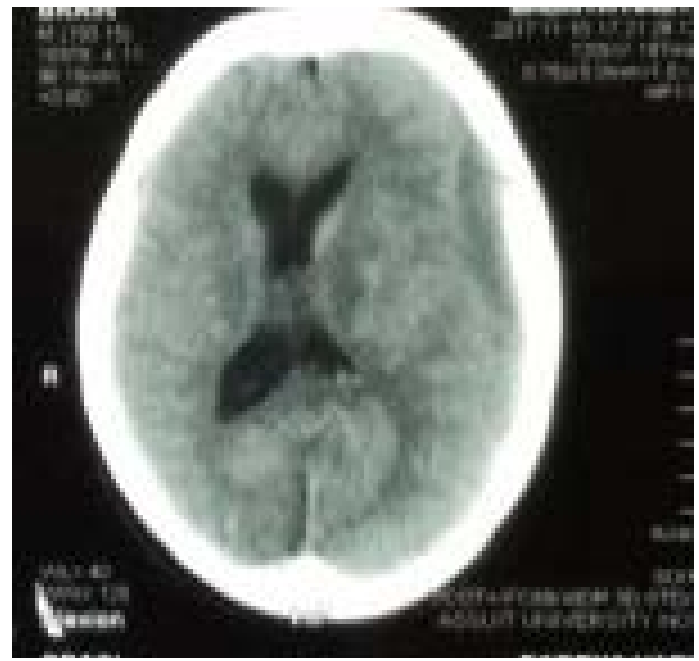

Figure 4. Showing left frontoparieal CSDH in a female patient 34 years old who was on marivan therapy following mitral valve replacement. 


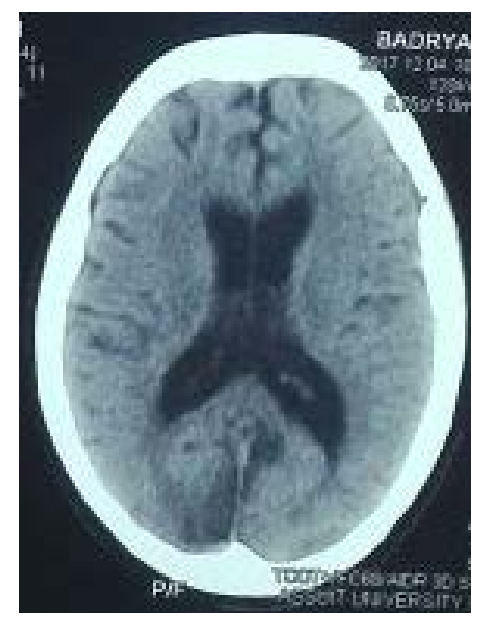

Figure 5. Showing complete evacuation of the left frontoparieal CSDH after using double twist drill craniostomy.

Grade 1: Alert and orientated: absence of mild symptoms such as headache, or mild neurological deficit such as reflex asymmetry.

Grade 2: Drowsy or disorientated, or variable neurological deficit such as hemiparesis.

Grade 3: Stuporous, but responding appropriately to noxious stimuli, several focal signs such as hemiplegia.

Grade 4: Comatose with absent motor responses to painful stimuli, decerebrate or decorticate posturing.

- Morbidity and mortality:

Morbidity in our study is defined as any complication resulted in symptoms or unpredicted intervention within 30 days, while mortality is defined as any death reported between surgery and discharge from hospital [7] [8].

\section{- Hospital stay:}

All the patients were admitted to the neurosurgery department on the day of the surgery or one day prior to it. The patient is discharged from the hospital to home after achievement of the aimed improvement. The patient can be discharged from the neurosurgical department to another department in the hospital if needed.

\section{- Postoperative complications:}

Including residual collection, postoperative seizures, pneumocephaly, subarachnoid hemorrhage, failure of cerebral re-expansion, recurrence, brain penetration, and wound infection.

\section{- Recurrence:}

Recurrence is defined in our study as a subdural collection in the same site of the operation which affects the improvement of the patient and indicates surgical re-evacuation [9].

\section{Results}

The study was performed on total of 21 patients. Eleven males (52.4\%) and ten 
females (47.6\%). Their age ranged from 9 days to 90 years, $(71.4 \%)$ of the patients were above 40 years and (28.6\%) were younger than 40 years.

Symptoms of the studied patients varied as $76.2 \%$ of the patients were presented by disturbed conscious level (DCL), $47.6 \%$ presented by weakness on the contralateral side of the hematoma, $19 \%$ presented by fits, $14.3 \%$ suffered from headache and $14.3 \%$ presented by dysphasia (Table 1 ).

The patients in this study had variable co-morbidities which is common in patients with chronic subdural hematoma which make these patients poor surgical candidates as shown in (Table 2).

Only 16 patients $(76 \%)$ among the studied patients were presented by disturbed conscious level, the other 5 patients (24\%) were fully conscious prior to the surgical procedure. the preoperative GCS ranged from 7 to 15.15 patients were improved consciously (93.75\%), while only 1 (6.25\%) patient died within the first few hours postoperative (Figure 6).

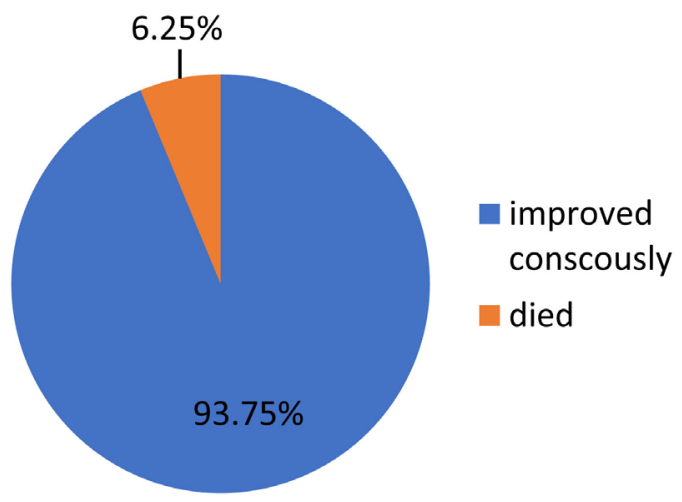

Figure 6. Conscious level improvement among preoperative disturbed level patients.

Table 1. The main complaints of the studied patients.

\begin{tabular}{ccc}
\hline Complaint & No. & $\%$ \\
\hline DCL & 16 & 76.2 \\
Weakness & 10 & 47.6 \\
Fits & 4 & 19 \\
Headache & 3 & 14.3 \\
Dysphasia & 3 & 14.3 \\
\hline
\end{tabular}

Table 2. Distribution of medical co-morbidities among the studied patients.

\begin{tabular}{ccc}
\hline Risk factors & No. (21) & $\%$ \\
\hline Cardiac disease & 8 & 38.1 \\
Coagulopathy & 7 & 33.3 \\
Blood thinners & 4 & 19.0 \\
Hepatic disease & 4 & 19.0 \\
Hypertension & 5 & 23.8 \\
\hline
\end{tabular}


The study included 18 (86\%) patients presented by motor weakness on the contralateral side of the hematoma while the other 3 (14\%) patients were full motor power on preoperative examination. Thirteen (13) patients showed postoperative complete improvement (full motor power ) (72.2\%), 4 patients showed incomplete improvement of the motor power prior to the discharge day (grade 4 by neurological examination) (22.2\%) 3 of them improved later on, and 1 patient did not improve and died postoperatively (5.5\%) (Figure 7).

Complications and postoperative follow up:

In this study 21 patients were operated using twist drill, 4 patients showed residual collection in the post-operative CT (19\%). Subarachnoid hemorrhage was detected in one patient proved by the postoperative CT (4.7\%). Pneumocephaly was detected in 2 patients (9.5\%). postoperative seizures occurred in 2 patients (9.5\%) (Table 3).

The postoperative follow up of our patients showed improvement of $20 \mathrm{pa}-$ tients (95.2\%), and the death of 1 patient (4.7\%).

The operative time was less than 10 minutes in $71.4 \%$ of the cases and ranged from 10 to 15 in $23.8 \%$ of them and only one case (4.7\%) lasted about $17 \mathrm{mi}-$ nutes (Table 4).

In our study 11 out of 21 patients were discharged before their $3^{\text {rd }}$ post-operative day, while 6 patients were discharged on the $3^{\text {rd }}$ day post-operative and 4 patients were discharged after their $3^{\text {rd }}$ day post-operative (Table 5 ).

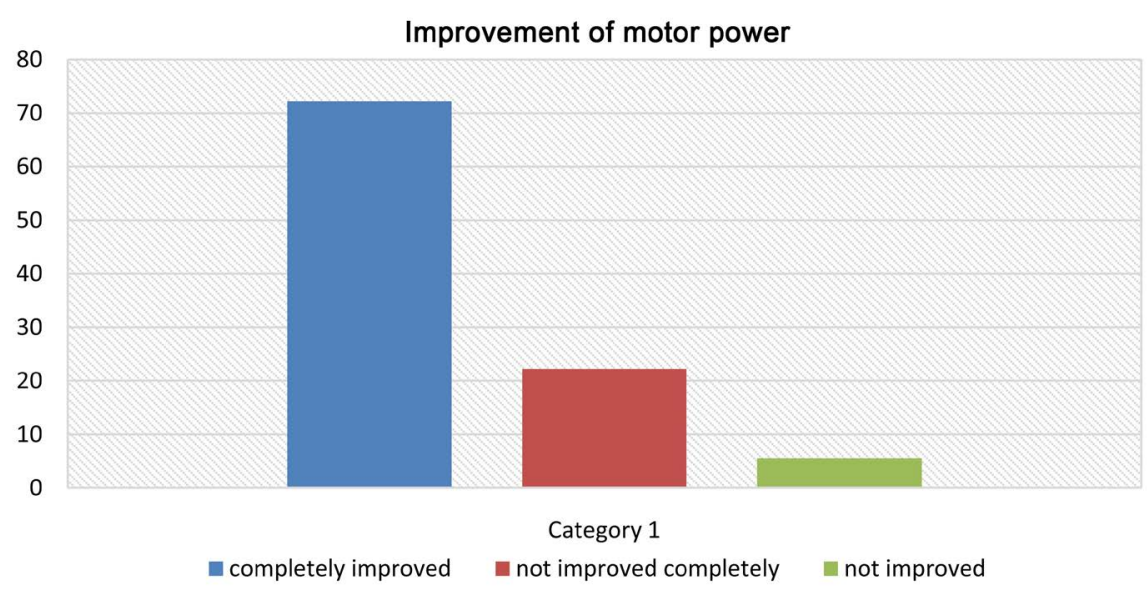

Figure 7. Improvement of motor power.

Table 3. Postoperative complications.

\begin{tabular}{ccc}
\hline Post-operative radiology & No. $(21)$ & $\%$ \\
\hline Residual collection & 4 & $19 \%$ \\
Pneumocephaly & 2 & $9.5 \%$ \\
Postoperative seizures & 2 & $9.5 \%$ \\
SAH & 1 & $4.7 \%$ \\
Total complicated patients & 5 & $23.8 \%$ \\
\hline
\end{tabular}


Table 4. Duration of operation.

\begin{tabular}{ccc}
\hline Duration of operation (min) & No. $(21)$ & $\%$ \\
\hline$<10$ & 15 & 71.4 \\
$10-15$ & 5 & 23.8 \\
$\geq 15$ & 1 & 4.7 \\
Mean \pm SD (Range) & & $8.38 \pm 3.33(6.0-17.0)$ \\
\hline
\end{tabular}

Table 5. Duration of hospital stay.

\begin{tabular}{ccc}
\hline Hospital stay: (days) & No. $(21)$ & $\%$ \\
\hline$<3$ days & 11 & 52.4 \\
3 days & 6 & 28.6 \\
$>3$ days & 4 & 19 \\
Mean \pm SD (Range) & & $3.3 \pm 1.34(2.0-7.0)$ \\
\hline
\end{tabular}

\section{Discussion}

With the increase of traumatic accidents and the prolongation of life expectancy, CSDHs are encountered much more frequently in aged population. In general, the mean age in some large investigations ranges from 56 to 63 years. In our series, it ranged from 9 days to 90 years with a mean of (57 years) consistent with other such studies by LIU Yuan et al. the number of female patients was smaller than that of male patients, close to the data of Sambasivan's [10] [11].

The differences with regard to genders and ages may relate to the more exposure of males to injury, the longer life expectancy for females, the lower requires for medical advices, and the protective effects of estrogen or its derivatives on capillaries [11] [12].

Head trauma has been identified as single most common factor associated with $25 \%-75 \%$ cases of CSDH in most of the studies. In our study, history of head trauma was present in $57 \%$ of cases. Other common causes in this study included cardiac diseases, coagulopathy, hypertension, hepatic diseases and using blood thinners [13] [14] [15] [16] [17].

According to Santarius et al., the most frequent presenting symptoms are gait abnormalities (57\%), disturbed conscious level (35\%), hemiparesis (35\%), and headache $(18 \%)$. In our series, the most common clinical findings were disturbed conscious level $(76.2 \%)$ in 16 cases of the studied patients, motor weakness in the contralateral side of the hematoma (47.6\% ), seizures (19\%), headache (14.3\%) and dysphasia [18].

\subsection{Primary Outcome: Operation Time}

Our results showed that the mean operative time (the time starts with the first surgical step and stops by its end) in our studied cases was 8.38 minutes with a mean operative room time (the time between the patient's entrance to the opera- 
tive theatre and his exit from it) approximately 32 minutes. Lee SJ, Hwang on 2016 in a study on twist drill evacuation of CSDH reported an operative time of 8.9 minutes which is close to our results [19].

Regan JM et al. on 2015 reported a mean operative room time in surgical evacuation of CSDH in methods other than twist drill evacuation estimated as 78.8 minutes [20].

According to the above-mentioned results, our primary aim of decreasing the operative time was accomplished.

\subsection{Secondary Outcomes}

\section{Improvement of conscious level and functional outcome}

In this study 16 patients of the studied 21 patients presented by impairment of the conscious level (76.2\%) 15 of these patients showed dramatic improvement of conscious level after evacuation of the hematoma with improvement result (93.75\%), in a study by Xin Lin comparing the evacuation of CSDH by twist drill vs. burr hole craniotomy the results of improvement were ( $88.8 \%$ vs. $75.5 \%)$ respectively [21].

Also, the outcome of our study included the assessment of improvement of the clinical performance of the patients using Markwalders Grading Scale, a lower grade on this scale indicates a better cognitive function. Hence a preoperative mean of 2.2 was lowered to 0.5 after twist drill evacuation (lowered by 1.7) indicating obvious improvement of the clinical performance and cognitive function of the studied patients. Ernestus RI. et al. reported lowering of the mean of MGS from 1.9 to 0.92 after evacuation of chronic subdural hematoma using burr hole craniostomy. Kim GH. et al. described in his study on 48 patients operated using twist drill that the preoperative mean of MGS was 1.29, which was lowered postoperatively to 0.25 (lowered by 1.04). And this difference between this study and our results is most probably due to the high percentage of patients of preoperatively low grades $(0,1$ and 2$)$ on MGS which was $93.75 \%$ of the patients in comparison to $58.4 \%$ in our study [11] [22].

\subsection{Lowering Morbidity}

Smely et al. compared a prospective series of CSDH patients and concluded that twist drill craniostomy is significantly better in outcome than burr hole craniostomy in lowering morbidity ( $0 \%$ vs. $18 \%$ ). Similarly in our study we used TDC on 14 patients (66\%) who had had variable medical co-morbidities (Table 2) which made them poor surgical candidates and elevated the risk of the surgical and anesthetic complications and yet the postoperative morbidity rate was $0 \%$ [7].

\subsection{Reducing Hospital Stay}

In our study the hospitalization period ranged from 2 days to 7 days with a mean of 3.3 days. According to the results reported by Smely et al. in their retrospec- 
tive BHE series a mean hospital stay of 9.6 days and 4.9 days in their prospective TDE series. Also in the work of Goyal RK, Nayak B et al. on 2018, TDE seems to be better than BHE in terms of duration of hospital stay (7.4 vs. 8.05). However, these differences were not statistically significant [7] [23].

\subsection{Post-Operative Complications}

Postoperative complications were noticed in 5 patients $(23.8 \%)$ in our study as following:

\section{Residual collection}

The most frequent complication was residual collection noticed in the postoperative CT in 4 patients (19\%), although the patients improved clinically and the patients returned to their normal life style within one month postoperative. And by clinical follow up to 6 months after the surgery, the patients continued their daily activities. A study by Jaiswal S. et al. showed residual collection in (30.56\%) of the patients after burr hole drainage in comparison to $34.15 \%)$ of the patients after twist drill craniostomy and this was however statistically insignificant. The reported incidence of residual collection after twist drill evacuation of $\mathrm{cSDH}$ in the literature was from $34 \%$ to $78 \%$, which is not correlated with the clinical outcome or recurrence of the hematoma but occurs due to the slow and steady re-expansion of the brain that normally occur after twist drill evacuation and usually spontaneously resolve within 40 days [10] [11] [24] [25].

\subsection{Seizures}

Seizure is also one of the surgical complications of evacuation of cSDH in our study it occurred in 2 patient (9.4\%) and this is higher than the results of Jaiswal et al. in a study on 41 patients underwent evacuation of $\mathrm{CSDH}$ using TDC which had no cases of postoperative seizures. while in the work of Rohde V et al. on 376 patients operated using BHC (13.6\%) exhibited postoperative seizures [24] [26].

\subsection{Pneumocephaly}

Pneumocephaly was found in 2 patients in our study (9.5\%). In the study of Wang QF. et al. on surgical treatment of cSDH Pneumocephaly was found in $86.6 \%$ of the cases operated using burr hole craniostomy and in $13 \%$ of the patients operated by twist drill craniostomy which was statistically significant [3] [27].

\subsection{Subarachnoid Hemorrhage}

Subarachnoid hemorrhage (SAH) is a rare surgical complication of cSDH evacuation. It was detected in one patient in our study, and this is thought to be due to the fragility of the blood vessels as the patient was 90 years old, with the presence of hypertension, a case report study by Seung WB and Jeong JH. Detected subarachnoid hemorrhage in a patient after burr hole craniotomy [28] [29]. 
No studies reported SAH after twist drill technique as it is a rare complication.

\subsection{Recurrence}

Following Goyal RK, Nayak B et al. who defined recurrence as the need for surgical re-evacuation in a study published on 2018, there were no cases of recurrence in our study but there were 4 cases (19\%) with residual hematoma which did not affect the patients' improvement and hence did not require surgical re-evacuation [9].

\subsection{Mortality Rate}

In our study the mortality rate was $4.7 \%$ (only one patient). The patient was 90 years old postoperatively she developed seizures, CT brain showed diffuse SAH and the patient died within 2 days. On a study by Weigel R. et al. the mortality rate in BHC group reached $32 \%$. The mortality rate in TDC group was significantly less than BHC group (8\%), which agree with our results mentioned above [8].

\subsection{Other Complications}

There are some other complications described in the literature such as intracerebral hemorrhage which may indicate surgical interference, brain penetration and wound infection or subdural empyema. None of these complications occurred in our study.

\section{Conclusions}

Twist drill technique is a safe and effective method in evacuation of $\mathrm{cSDH}$ and can be considered the first-choice treatment in cases with poor surgical candidates and patients with comorbidities.

Twist drill craniostomy is superior to burr hole craniostomy as:

Twist drill is less invasive maneuver with small wound and minimal scarring, short operative time, and short hospital stay. It can be easily done with local anesthesia, less morbidity and mortality and feasibility to be done in poor surgical candidates for general anesthesia.

\section{Recommendations}

We recommend studying double twist drill craniostomy technique in comorbid patients in comparison to burr hole craniostomy and on a larger number of patients.

\section{Limitations of the Study}

Our study examined small sample size with a relatively a weak potential to represent the people suffering from $\mathrm{CSDH}$, so further randomized researches on a larger population is required to provide more informative data about the value of double twist drill craniostomy. 


\section{Conflicts of Interest}

The authors declare no conflicts of interest regarding the publication of this paper.

\section{References}

[1] Gelabert-Gonzáles, M., Iglesias-Pais, M., García-Allut, A. and Martínez-Rumbo, R. (2005) Chronic Subdural Haematoma: Surgical Treatment and Outcome in 1000 Cases. Clinical Neurology and Neurosurgery, 107, 223-229. https://doi.org/10.1016/j.clineuro.2004.09.015

[2] Yamamoto, H., Hirashima, Y., Hamada, H., Hayashi, N., Origasa, H. and Endo, S. (2003) Independent Predictors of Recurrence of Chronic Subdural Hematoma: Results of Multivariate Analysis Performed Using a Logistic Regression Model. Journal of Neurosurgery, 98, 1217-1221. https://doi.org/10.3171/jns.2003.98.6.1217

[3] Yadav, Y.R., Parihar, V., Namdev, H. and Bajaj, J. (2016) Chronic Subdural Hematoma. Asian Journal of Neurosurgery, 11, 330. https://doi.org/10.4103/1793-5482.145102

[4] Choy, E.H. and Panayi, G.S. (2001) Cytokine Pathways and Joint Inflammation in Rheumatoid Arthritis. The New England Journal of Medicine, 344, 907-916. https://doi.org/10.1056/NEJM200103223441207

[5] Yadav, Y.R., Yadav, S. and Parihar, V. (2013) Modified Twist Drill Technique in the Management of Chronic Subdural Hematoma. Turkish Neurosurgery, 23, 50-54. https://doi.org/10.5137/1019-5149.JTN.6655-12.1

[6] Lin, X. (2011) Comparing Twist-Drill Drainage with Burr Hole Drainage for Chronic Subdural Hematoma. Chinese Journal of Traumatology, 14, 170-173.

[7] Smely, C., Madlinger, A. and Scheremet, R. (1997) Chronic Subdural Haematoma-A Comparison of Two Different Treatment Modalities. Acta Neurochirurgica (Wien), 139, 818-825. https://doi.org/10.1007/BF01411399

[8] Weigel, R., Schmiedek, P. and Krauss, J.K. (2003) Outcome of Contemporary Surgery for Chronic Subdural Haematoma: Evidence Based Review. Journal of Neurology, Neurosurgery, and Psychiatry, 74, 937-943.

https://doi.org/10.1136/jnnp.74.7.937

[9] Goyal, R.K., Nayak, B., et al. (2018) Management of Chronic Subdural Hematoma: Burr Hole versus Twist Drill-A Prospective Study. Asian Journal of Neurosurgery, 13, 319. https://doi.org/10.4103/ajns.AJNS_99_16

[10] Sambavian, M. (1997) An Overview of Chronic Subdural Hematoma: Experience with 2300 Cases. Surgical Neurology, 47, 418-422. https://doi.org/10.1016/S0090-3019(97)00188-2

[11] Ernestus, R.I., Beldzinski, P., Lanfermann, H., et al. (1997) Chronic Subdural Haematoma: Surgical Treatment and Outcome in 104 Patients. Surgical Neurology, 48, 220-225. https://doi.org/10.1016/S0090-3019(97)80031-6

[12] Giuffrè, R., Palma, E., Liccardo, G., et al. (1992) Sex Steroid Hormones in the Pathogenesis of Chronic Subdural Hematoma. Neurochirurgia (Stuttg), 35, 103-107. https://doi.org/10.1055/s-2008-1052258

[13] Han, S.B., Choi, S.W., Song, S.H., Youm, J.Y., Koh, H.S., Kim, S.H. and Kwon, H.J. (2014) Prediction of Chronic Subdural Hematoma in Minor Head Trauma Patients. Korean Journal of Neurotrauma, 10, 106-111. https://doi.org/10.13004/kjnt.2014.10.2.106 
[14] Trotter, W. (1914) Chronic Subdural Haemorrhage of Traumatic Origin, and Its Relation to Pachymeningitis Haemorrhagica Interna. British Journal of Surgery, 2, 271-291. https://doi.org/10.1002/bjs.1800020608

[15] Naffziger, H.C. (1924) Subdural Fluid Accumulations Following Head Injury. JAMA, 82, 1751-1758. https://doi.org/10.1001/jama.1924.02650480007004

[16] Kudo, H., Kuwamura, K., Izawa, I., Sawa, H. and Tamaki, N. (1992) Chronic Subdural Hematoma in Elderly People: Present Status on Awaji Island and Epidemiological Prospect. Neurologia Medico-Chirurgica (Tokyo), 32, 207-209. https://doi.org/10.2176/nmc.32.207

[17] Baechli, H., Nordmann, A., Bucher, H.C. and Gratzl, O. (2004) Demographics and Prevalent Risk Factors of Chronic Subdural Haematoma: Results of a Large Single-Center Cohort Study. Neurosurgical Review, 27, 263-266. https://doi.org/10.1007/s10143-004-0337-6

[18] Santarius, T., et al. (2009) Use of Drains versus No Drains after Burr-Hole Evacuation of Chronic Subdural Haematoma: A Randomised Controlled Trial. The Lancet, 374, 1067-1073. https://doi.org/10.1016/S0140-6736(09)61115-6

[19] Lee, S.J., Hwang, S.C. and Im, S.B. (2016) Twist-Drill or Burr Hole Craniostomy for Draining Chronic Subdural Hematomas: How to Choose It for Chronic Subdural Hematoma Drainage. Korean Journal of Neurotrauma, 12, 107-111. https://doi.org/10.13004/kjnt.2016.12.2.107

[20] Regan, J.M., Worley, E., et al. (2015) Burr Hole Washout versus Craniotomy for Chronic Subdural Hematoma: Patient Outcome and Cost Analysis. PLoS ONE, 10, e0115085. https://doi.org/10.1371/journal.pone.0115085

[21] Xin, L. (2011) Comparing Twist-Drill Drainage with Burr Hole Drainage for Chronic Subdural Hematoma. Chinese Journal of Traumatology (English Edition), 14, 170-173.

[22] Kim, G.H., et al. (2014) Comparison of the Indications and Treatment Results of Burr-Hole Drainage at the Maximal Thickness Area versus Twist-Drill Craniostomy at the Pre-Coronal Point for the Evacuation of Symptomatic Chronic Subdural Hematomas. Journal of Korean Neurosurgical Society, 56, 243. https://doi.org/10.3340/jkns.2014.56.3.243

[23] Markwalder, T.M. (2000) The Course of Chronic Subdural Hematomas after Burr-Hole Craniostomy with and without Closed-System Drainage. Neurosurgery Clinics, 11, 541-546. https://doi.org/10.1016/S1042-3680(18)30120-7

[24] Jaiswal, S., et al. (2017) Twist Drill Craniostomy vs Burr Hole Craniostomy in Chronic Subdural Hematoma: A Randomized Study. Romanian Neurosurgery, 31, 346-355. https://doi.org/10.1515/romneu-2017-0057

[25] Berhouma, M. and Krolak-Salmon, P. (2017) Brain and Spine Surgery in the Elderly. Springer, Berlin. https://doi.org/10.1007/978-3-319-40232-1

[26] Rohde, V., Graf, G. and Hassler, W. (2002) Complications of Burr-Hole Craniostomy and Closed-System Drainage for Chronic Subdural Hematomas: A Retrospective Analysis of 376 Patients. Neurosurgical Review, 25, 89-94. https://doi.org/10.1007/s101430100182

[27] Wang, Q.F., Cheng, C. and You, C. (2016) A New Modified Twist Drill Craniostomy Using a Novel Device to Evacuate Chronic Subdural Hematoma. Medicine, 95, e3036. https://doi.org/10.1097/MD.0000000000003036

[28] Rusconi, A., et al. (2015) Infrequent Hemorrhagic Complications Following Surgical Drainage of Chronic Subdural Hematomas. Journal of Korean Neurosurgical So- 
ciety, 57, 379. https://doi.org/10.3340/jkns.2015.57.5.379

[29] Seung, W.B. and Jeong, J.H. (2017) Postoperative Subarachnoid Hemorrhage and Multipunctate Intracerebral Hemorrhages Following Evacuation of Bilateral Chronic Subdural Hematomas. Korean Journal of Neurotrauma, 13, 149-152.

https://doi.org/10.13004/kjnt.2017.13.2.149 